\title{
Modified Relaxed Extragradient Method for a General System of Variational Inequalities and Nonexpansive Mappings in Banach Spaces
}

\author{
Yuanheng Wang and Liu Yang \\ Department of Mathematics, Zhejiang Normal University, Zhejiang 321004, China \\ Correspondence should be addressed to Yuanheng Wang,wangyuanheng@yahoo.com.cn
}

Received 15 May 2012; Accepted 8 June 2012

Academic Editor: Yonghong Yao

Copyright (c) 2012 Y. Wang and L. Yang. This is an open access article distributed under the Creative Commons Attribution License, which permits unrestricted use, distribution, and reproduction in any medium, provided the original work is properly cited.

The purpose of this paper is to introduce a new modified relaxed extragradient method and study for finding some common solutions for a general system of variational inequalities with inversestrongly monotone mappings and nonexpansive mappings in the framework of real Banach spaces. By using the demiclosedness principle, it is proved that the iterative sequence defined by the relaxed extragradient method converges strongly to a common solution for the system of variational inequalities and nonexpansive mappings under quite mild conditions.

\section{Introduction}

Let $H$ be a real Hilbert space with inner product $\langle\cdot, \cdot\rangle$ and norm $\|\cdot\|$, and $C$ be a nonempty closed convex subset of $H$. Let $P_{C}$ be the projection of $H$ onto $C$, it is known that projection operator $P_{C}$ is nonexpansive and satisfies the following:

$$
\left\langle x-y, P_{C} x-P_{C} y\right\rangle \geq\left\|P_{C} x-P_{C} y\right\|^{2}, \quad \forall x, y \in H .
$$

Moreover, $P_{C} x$ is characterized by the properties $P_{C} x \in C$ and $\left\langle x-P_{C} x, P_{C} x-y\right\rangle \geq 0$ for all $y \in C$.

Let $A: C \rightarrow H$ be a mapping. Recall that the classical variational inequality, denoted by $\operatorname{VI}(C, A)$, is to find $u \in V$ such that

$$
\langle A u, v-u\rangle \geq 0, \quad \forall v \in C
$$


One can see that the variational inequality (1.2) is equivalent to a fixed point problem. $A$ element $x^{*} \in C$ is a solution of the variational inequality (1.2) if and only if $x^{*} \in C$ is a fixed point of the mapping $P_{C}(I-\lambda A)$, where $I$ is the identity mapping and $\lambda>0$ is a constant.

Variational inequalities introduced by Stampacchia [1] in the early sixties have had a great impact and influence in the development of almost all branches of pure and applied sciences and have witnessed an explosive growth in theoretical advances, algorithmic development, and so forth; see, for example, [1-18] and the references therein.

For a monotone mapping $A: C \rightarrow H$, Noor [2] studied the following problem of finding $\left(x^{*} ; y^{*}\right) \in C \times C$ such that:

$$
\begin{array}{ll}
\left\langle\lambda A y^{*}+x^{*}-y^{*}, x-x^{*}\right\rangle \geq 0, & \forall x \in C, \\
\left\langle\mu A x^{*}+y^{*}-x^{*}, x-y^{*}\right\rangle \geq 0, & \forall x \in C,
\end{array}
$$

where $\lambda, \mu>0$ are constants. If we add up the requirement that $x^{*}=y^{*}$, then the problem (1.3) is reduced to the classical variational inequality (1.2). The problem of finding solutions of (1.3) by using iterative methods has been studied by many authors; see [2-9] and the references therein.

Recently, some authors also studied the problem of finding a common element of the fixed point set of nonexpansive mappings and the solution set of variational inequalities for $\alpha$ inversestrongly monotone mappings in the framework of real Hilbert spaces [10] and Banach spaces [11].

On the other hands, Ceng et al. [12] introduce the following general system of variational inequalities involving two different operators. For two given operators, consider the problem finding $x^{*}, y^{*} \in C$ such that

$$
\begin{array}{ll}
\left\langle\lambda A y^{*}+x^{*}-y^{*}, x-x^{*}\right\rangle \geq 0, & \forall x \in C, \\
\left\langle\mu B x^{*}+y^{*}-x^{*}, x-y^{*}\right\rangle \geq 0, & \forall x \in C,
\end{array}
$$

where $\lambda, \mu>0$ are constants. To illustrate the applications of this system, we can refer to an example of related nonlinear optimization problem put forward by Zhu and Marcotte [13]. Very recently, Yao et al. [14] extend the system of variational inequality problems (1.4) to Banach spaces.

In the present paper, motivated and inspired by the methods of Ceng et al. [12], Iiduka and Takahashi [10], Qin et al. [11], and Yao et al. [14], we consider the following general system of variational inequalities in Banach spaces.

Let $C$ be a nonempty closed convex subset of a real smooth Banach space. Let $A, B$ : $C \rightarrow E$ be $\alpha$-inversestrongly accretive mapping and $\beta$-inverse-strongly accretive mapping. Find $\left(x^{*}, y^{*}\right) \in C \times C$ such that

$$
\begin{aligned}
& \left\langle\lambda A y^{*}+x^{*}-y^{*}, j\left(x-x^{*}\right)\right\rangle \geq 0, \quad \forall x \in C, \\
& \left\langle\mu B x^{*}+y^{*}-x^{*}, j\left(x-y^{*}\right)\right\rangle \geq 0, \quad \forall x \in C,
\end{aligned}
$$

where $\lambda, \mu>0$ are constants, and $j$ is the normalized duality mapping. For more details of $j$, one may see $\mathrm{Li}[15]$. In a real Hilbert space, $j=I$ is the identity mapping, and the system 
(1.5) is reduced to (1.4). If we add up the requirement that $A=B$, then the problem (1.4) is reduced to the generalized variational inequality (1.3), in particular.

And we consider the problem of finding a common element of the fixed point set of nonexpansive mappings and the solution set of the general system of variational inequalities for $\alpha$-inversestrongly monotone mappings in the framework of real Banach spaces. By using the demiclosedness principle, we prove that under quite mild conditions the iterative sequence defined by the relaxed extragradient method converges strongly to a common solution of this system of variational inequalities and nonexpansive mappings. Our results improve and extend the corresponding results announced by other authors, such as [2$4,6,8,10-12,14]$.

\section{Preliminaries}

Let $C$ be a nonempty closed convex subset of a Banach space of $E$. Let $E^{*}$ be the dual space of $E$, and let $\langle\cdot, \cdot\rangle$ denote the pairing between $E$ and $E^{*}$. For $q>1$, the generalized duality mapping $J_{q}: E \rightarrow 2^{E^{*}}$ is defined by

$$
J_{q}(x)=\left\{f \in E^{*}:\langle x, f\rangle=\|x\|^{q},\|f\|=\|x\|^{q}\right\},
$$

for all $x \in E$. In particular, $J=J_{2}$ is called the normalized duality mapping. It is known that $J_{q}(x)=\|x\|^{q-2} J(x)$ for all $x \in E$. If $E$ is a Hilbert space, then $J=I$ is the identity mapping. Further, we have the following properties of the generalized duality mapping $J_{q}$ :

(1) $J_{q}(x)=\|x\|^{q-2} J_{2}(x)$ for all $x \in E$ with $x \neq 0$,

(2) $J_{q}(t x)=t^{q-1} J_{q}(x)$ for all $x \in E$ and $t \in[0, \infty)$,

(3) $J_{q}(-x)=-J_{q}(x)$ for all $x \in E$.

Let $U=\{x \in E:\|x\|=1\}$. $E$ is said to be uniformly convex if, for any $\epsilon \in(0,2]$, there exists $\delta>0$ such that for any $x, y \in U$. $\|x-y\| \geq \epsilon$ implies $\|(x+y) / 2\| \leq(1-\delta)$.

It is known that a uniformly convex Banach space is reflexive and strictly convex, $E$ is said to be Gâteaux differentiable if the limit

$$
\operatorname{Lim}_{t \rightarrow 0} \frac{\|x+t y\|-\|x\|}{t}
$$

exists for each $x, y \in U$. In this case, $E$ is said to be smooth. The norm of $E$ is said to be uniformly Gâteaux differentiable if, for each $y \in U$, the limit (2.2) is attained uniformly for $x \in U$. The norm of $E$ is said to be Frêchet differentiable, if, for each $x \in U$, the limit (2.2) is attained uniformly for $y \in U$. The norm of $E$ is said to be uniformly Frêchet differentiable if the limit (2.2) is attained uniformly for $x, y \in U$. It is well-known that (uniform) Frêchet differentiable of the norm of $E$ implies (uniform) Gâteaux differentiability of the norm of $E$.

The modulus of smoothness of $E$ is defined by

$$
\rho(\tau)=\sup \left\{\frac{1}{2}(\|x+y\|+\|x-y\|)-1: x, y \in E,\|x\|=1,\|y\| \leq t\right\},
$$

where $\rho:[0, \infty) \rightarrow[0, \infty)$ is a function. It is known that a Banach space $E$ is uniformly smooth if and only if $\lim _{(n \rightarrow \infty)}(\rho(t) / t)=0$. Let $q$ be a fixed real number with $1<q \leq 2$. 
A Banach space $E$ is said to be $q$ uniformly smooth if there exists a fixed constant $c>0$ such that $\rho(t) \leq c t^{q}$, for all $t>0$.

Next, we always assume that $E$ is a smooth Banach space. Let $C$ be a nonempty closed convex subsets of $E$. Recall that an operator $A$ of $C$ into $E$ is said to be accretive if

$$
\langle A x-A y, j(x-y)\rangle \geq 0, \quad \forall x, y \in C .
$$

$A$ is said to be $\alpha$-inversestrongly accretive if there exists a constant $\alpha>0$ such that

$$
\langle A x-A y, j(x-y)\rangle \geq \alpha\|A x-A y\|^{2}, \quad \forall x, y \in C .
$$

Let $D$ be a subset of $C$ and $Q$ be a mapping of $C$ into $D$. Then $Q$ is said to be sunny if

$$
Q(Q x+t(x-Q x))=Q x
$$

whenever $Q x+t(x-Q x) \in C$ for $x \in C$ and $t \geq 0$. A subset $D$ of $C$ is called a sunny nonexpansive retract of $C$ if there exists a sunny nonexpansive retraction from $C$ onto $D$.

In order to prove the main result, we also need the following lemmas. The following Lemma 2.2 describes characterization of sunny nonexpansive retraction on a smooth Banach space.

Lemma 2.1 (see [16]). Let E be a real 2 uniformly smooth Banach space with the best smooth constant $K$. Then the following inequality holds:

$$
\|x+y\|^{2} \leq\|x\|^{2}+2\langle y, j x\rangle+2\|K y\|^{2}, \quad \forall x, y \in E .
$$

Lemma 2.2 (see [17]). Let $C$ be a closed convex subset of a smooth Banach space E, let $D$ be a nonempty subset of $C$, and let $Q$ be a retraction form $C$ onto $D$. Then $Q$ is sunny and nonexpansive if and only if

$$
\langle u-Q u, j(y-Q u)\rangle \leq 0
$$

for all $u \in C$ and $y \in D$.

Lemma 2.3 (see [18]). Let $\left\{x_{n}\right\}$ and $\left\{y_{n}\right\}$ be bounded sequences in a Banach space $E$ and a sequence $\left\{\beta_{n}\right\}$ in $[0,1]$ with

$$
0<\liminf _{n \rightarrow \infty} \beta_{n} \leq \limsup _{n \rightarrow \infty} \beta_{n}<1
$$

Suppose that $x_{n+1}=\left(1-\beta_{n}\right) y_{n}+\beta_{n} x_{n}$ for all integers $n \geq 0$ and

$$
\limsup _{n \rightarrow \infty}\left(\left\|y_{n+1}-y_{n}\right\|-\left\|x_{n+1}-x_{n}\right\|\right) \leq 0
$$

Then, $\lim _{(n \rightarrow \infty)}\left\|y_{n}-x_{n}\right\|=0$. 
Lemma 2.4 (see [19]). Let $C$ be a nonempty closed convex subset of a real uniformly smooth Banach space. Let $S_{1}$ and $S_{2}$ be two nonexpansive mappings from $C$ into itself with a common fixed point. Define a mapping $S: C \rightarrow C$ by

$$
S x=\delta S_{1} x+(1-\delta) S_{2} x, \quad \forall x \in C
$$

where $\delta$ is a constant in $(0,1)$. Then $S$ is nonexpansive and $F(S)=F\left(S_{1}\right) \cap F\left(S_{2}\right)$.

Lemma 2.5 (see [20]). Assume that $\left\{\alpha_{n}\right\}$ is a sequence of nonnegative real numbers such that

$$
\alpha_{n+1} \leq\left(1-\gamma_{n}\right) \alpha_{n}+\delta_{n}
$$

where $\left\{\gamma_{n}\right\}$ is a sequence in $(0,1)$ and $\left\{\delta_{n}\right\}$ is a sequence such that

(a) $\sum_{n=1}^{\infty} \gamma_{n}=\infty$,

(b) $\lim _{(n \rightarrow \infty)}\left(\delta_{n} / \gamma_{n}\right) \leq 0$, or $\sum_{n=1}^{\infty}\left|\delta_{n}\right|<\infty$.

Then $\lim _{(n \rightarrow \infty)} \alpha_{n}=0$.

Lemma 2.6. Let $C$ be a nonempty closed convex subset of a real 2 uniformly smooth Banach space $E$ with the best smooth constant $K$. Let the mappings $A, B: C \rightarrow E$ be $\alpha$-inverse strongly accretive and $\beta$-inverse strongly accretive, respectively, and then one has $I-\lambda A$ and $I-\mu B$ are nonexpansive, where $\lambda \in\left(0, \alpha / K^{2}\right), \mu \in\left(0, \beta / K^{2}\right)$.

Proof. Indeed, for all $x, y \in C$, from Lemma 2.1, we have

$$
\begin{aligned}
\|(I-\lambda A) x-(I-\lambda A) y\|^{2} & =\|(x-y)-\lambda(A x-A y)\|^{2} \\
& \leq\|x-y\|^{2}-2 \lambda\langle A x-A y, j(x-y)\rangle+2 K^{2} \lambda^{2}\|A x-A y\|^{2} \\
& \leq\|x-y\|^{2}-2 \lambda \alpha\|A x-A y\|^{2}+2 K^{2} \lambda^{2}\|A x-A y\|^{2} \\
& \leq\|x-y\|^{2}+2 \lambda\left(K^{2} \lambda-\alpha\right)\|A x-A y\|^{2} \\
& \leq\|x-y\|^{2} .
\end{aligned}
$$

This shows that $I-\lambda A$ is nonexpansive mapping, so is $I-\mu B$.

Lemma 2.7. Let $C$ be a nonempty closed convex subset of a real 2 uniformly smooth Banach space $E$. Let $Q_{C}$ be the sunny nonexpansive retraction from $E$ onto $C$. Let $A, B: C \rightarrow E$ be $\alpha$-inverse strongly accretive mapping and $\beta$-inverse strongly accretive mapping, respectively. Let $G: C \rightarrow C$ be a mapping defined by

$$
G(x)=Q_{C}\left[Q_{C}(x-\mu B x)-\lambda A Q_{C}(x-\mu B x)\right], \quad \forall x \in C .
$$

If $\lambda \in\left(0, \alpha / K^{2}\right), \mu \in\left(0, \beta / K^{2}\right)$, then $G: C \rightarrow C$ is nonexpansive. 
Proof. For all $x, y \in C$, from Lemma 2.6, we have

$$
\begin{aligned}
\|G(x)-G(y)\|= & \| Q_{C}\left[Q_{C}(x-\mu B x)-\lambda A Q_{C}(x-\mu B x)\right] \\
& \quad-Q_{C}\left[Q_{C}(y-\mu B y)-\lambda A Q_{C}(y-\mu B y)\right] \| \\
\leq & \left\|Q_{C}(x-\mu B x)-\lambda A Q_{C}(x-\mu B x)-\left[Q_{C}(y-\mu B y)-\lambda A Q_{C}(y-\mu B y)\right]\right\| \\
= & \left\|(I-\lambda A) Q_{C}(I-\mu B) x-(I-\lambda A) Q_{C}(I-\mu B) y\right\| \\
\leq & \left\|Q_{C}(I-\mu B) x-Q_{C}(I-\mu B) y\right\| \\
\leq & \|(I-\mu B) x-(I-\mu B) y\| \\
\leq & \|x-y\| .
\end{aligned}
$$

Therefore, from (2.15), we obtain immediately that the mapping $G$ is nonexpansive.

Lemma 2.8. Let $C$ be a nonempty closed convex subset of a real smooth Banach space $E$. Let $Q_{C}$ be the sunny nonexpansive retraction from $E$ onto $C$. Let $A, B: C \rightarrow E$ be two possibly nonlinear mappings. For given $x^{*}, y^{*} \in C,\left(x^{*}, y^{*}\right)$ is a solution of problem (1.5) if and only if $x^{*}=Q_{C}\left(y^{*}-\lambda A y^{*}\right)$, where $y^{*}=Q_{C}\left(x^{*}-\mu B x^{*}\right)$.

Proof. We note that we can rewrite (1.5) as

$$
\begin{aligned}
& \left\langle x^{*}-\left(y^{*}-\lambda A y^{*}\right), j\left(x-x^{*}\right)\right\rangle \geq 0, \quad \forall x \in C, \\
& \left\langle y^{*}-\left(x^{*}-\lambda B x^{*}\right), j\left(x-y^{*}\right)\right\rangle \geq 0, \quad \forall x \in C .
\end{aligned}
$$

From Lemma 2.2, we can deduce that (2.16) is equivalent to

$$
\begin{aligned}
& x^{*}=Q_{C}\left(y^{*}-\lambda A y^{*}\right), \\
& y^{*}=Q_{C}\left(x^{*}-\mu B x^{*}\right) .
\end{aligned}
$$

This completes the proof.

Remark 2.9. From Lemma 2.8, we note that $x^{*}=Q_{C}\left[Q_{C}\left(x^{*}-\mu B x^{*}\right)-\lambda A Q_{C}\left(x^{*}-\mu B x^{*}\right)\right]$, which implies that $x^{*}$ is a fixed point of the mappings $G$.

\section{Main Result}

To solve the general system of variation inequality problem (1.5), now we are in a position to state and prove the main result in this paper.

Theorem 3.1. Let E be a uniformly convex and 2 uniformly smooth Banach space with the best smooth constant $K, C$ a nonempty closed convex subset of $E$, and $Q_{C}$ be the sunny nonexpansive retraction from $E$ onto $C$. Let $A, B: C \rightarrow E$ be $\alpha$-inverse strongly accretive mapping and $\beta$-inverse strongly 
accretive mapping, respectively, and $S: C \rightarrow C$ a nonexpansive mapping with a fixed point. Assume that $F=F(S) \cap F(G) \neq \emptyset$, where $G$ is defined as Lemma 2.7. Let $\left\{x_{n}\right\}$ be a sequence generated in the following manner:

$$
\begin{gathered}
x_{1}=u \in C, \\
y_{n}=Q_{C}\left(x_{n}-\mu B x_{n}\right), \\
x_{n+1}=\alpha_{n} u+\beta_{n} x_{n}+\gamma_{n}\left[\delta_{n} S x_{n}+\left(1-\delta_{n}\right) Q_{C}\left(y_{n}-\lambda A y_{n}\right)\right], \quad n \geq 1,
\end{gathered}
$$

where $\delta_{n} \subset[0,1], \lambda \in\left(0, \alpha / K^{2}\right), \mu \in\left(0, \beta / K^{2}\right)$, and $\left\{\alpha_{n}\right\},\left\{\beta_{n}\right\}$, and $\left\{\gamma_{n}\right\}$ are three sequences in $(0,1)$, and the following conditions are satisfied

(1) $\alpha_{n}+\beta_{n}+\gamma_{n}=1$, for all $n \geq 1$,

(2) $\lim _{(n \rightarrow \infty)} \alpha_{n}=0, \sum_{n=0}^{\infty} \alpha_{n}=\infty$,

(3) $0<\liminf _{(n \rightarrow \infty)} \beta_{n} \leq \lim \sup _{(n \rightarrow \infty)} \beta_{n}<1$,

(4) $\lim _{(n \rightarrow \infty)} \delta_{n}=\delta \in(0,1)$.

Then the sequence $\left\{x_{n}\right\}$ defined by (3.1) converges strongly to $\bar{x}=Q_{F} u$, and $(\bar{x}, \bar{y})$ is a solution of the problem (1.5), where $\bar{y}=Q_{C}(\bar{x}-\mu B \bar{x}), Q_{F}$ is a sunny nonexpansive retraction of $C$ onto $F$.

Proof. We divide the proof of Theorem 3.1 into six steps.

Step 1. First, we prove that $F$ is closed and convex. We know that $F(S)$ is closed and convex. Next, we show that $F(G)$ is closed and convex. From Lemma 3.1 and 3.2, we can see that $I-\lambda A, I-\mu B, G$ are nonexpansive. This shows that $F=F(S) \cap F(G)$ is closed and convex.

Step 2. Now we prove that the sequences $\left\{x_{n}\right\},\left\{y_{n}\right\},\left\{t_{n}\right\},\left\{A y_{n}\right\}$, and $\left\{B x_{n}\right\}$ are bounded. Let $x^{*} \in F(S) \cap F(G)$, and from Remark 2.9, we obtain that

$$
x^{*}=Q_{C}\left[Q_{C}\left(x^{*}-\mu B x^{*}\right)-\lambda A Q_{C}\left(x^{*}-\mu B x^{*}\right)\right] .
$$

Putting $y^{*}=Q_{C}\left(x^{*}-\mu B x^{*}\right)$, we see that

$$
x^{*}=Q_{C}\left(y^{*}-\lambda A y^{*}\right)
$$

Putting $t_{n}=\delta_{n} S x_{n}+\left(1-\delta_{n}\right) Q_{C}\left(y_{n}-\lambda A y_{n}\right)$ for each $n \geq 1$, we arrive at

$$
\begin{aligned}
\left\|t_{n}-x^{*}\right\| & =\left\|\delta_{n} S x_{n}+\left(1-\delta_{n}\right) Q_{C}\left(y_{n}-\lambda A y_{n}\right)-x^{*}\right\| \\
& \leq \delta_{n}\left\|S x_{n}-x^{*}\right\|+\left(1-\delta_{n}\right)\left\|Q_{C}\left(y_{n}-\lambda A y_{n}\right)-x^{*}\right\| \\
& \leq \delta_{n}\left\|x_{n}-x^{*}\right\|+\left(1-\delta_{n}\right)\left\|Q_{C}\left(y_{n}-\lambda A y_{n}\right)-Q_{C}\left(y^{*}-\lambda A y^{*}\right)\right\| \\
& \leq \delta_{n}\left\|x_{n}-x^{*}\right\|+\left(1-\delta_{n}\right)\left\|y_{n}-y^{*}\right\| \\
& =\delta_{n}\left\|x_{n}-x^{*}\right\|+\left(1-\delta_{n}\right)\left\|Q_{C}\left(x_{n}-\mu B x_{n}\right)-Q_{C}\left(x^{*}-\mu B x^{*}\right)\right\| \\
& \leq \delta_{n}\left\|x_{n}-x^{*}\right\|+\left(1-\delta_{n}\right)\left\|x_{n}-x^{*}\right\| \\
& =\left\|x_{n}-x^{*}\right\| .
\end{aligned}
$$


Hence, it follows that

$$
\begin{aligned}
\left\|x_{n+1}-x^{*}\right\| & =\left\|\alpha_{n} u+\beta_{n} x_{n}+\gamma_{n} t_{n}-x^{*}\right\| \\
& \leq \alpha_{n}\left\|u-x^{*}\right\|+\beta_{n}\left\|x_{n}-x^{*}\right\|+\gamma_{n}\left\|t_{n}-x^{*}\right\| \\
& \leq \alpha_{n}\left\|u-x^{*}\right\|+\beta_{n}\left\|x_{n}-x^{*}\right\|+\gamma_{n}\left\|x_{n}-x^{*}\right\| \\
& \leq \alpha_{n}\left\|u-x^{*}\right\|+\left(1-\alpha_{n}\right)\left\|x_{n}-x^{*}\right\| \\
& \leq \max \left\{\left\|x_{1}-x^{*}\right\|,\left\|u-x^{*}\right\|\right\} \\
& =\left\|u-x^{*}\right\| .
\end{aligned}
$$

Therefore, $\left\{x_{n}\right\}$ is bounded. Hence $\left\{x_{n}\right\},\left\{y_{n}\right\},\left\{t_{n}\right\},\left\{A y_{n}\right\}$, and $\left\{B x_{n}\right\}$ are bounded.

On the other hand, we have

$$
\begin{aligned}
\left\|t_{n+1}-t_{n}\right\|= & \| \delta_{n+1} S x_{n+1}+\left(1-\delta_{n+1}\right) Q_{C}\left(y_{n+1}-\lambda_{A} y_{n+1}\right)-\left(\delta_{n} S x_{n}\right) \\
& \quad+\left(1-\delta_{n}\right) Q_{C}\left(y_{n}-\lambda A y_{n}\right) \| \\
= & \| \delta_{n+1}\left(S x_{n+1}-S x_{n}\right)+\left(1-\delta_{n+1}\right)\left(Q_{C}\left(y_{n+1}-\lambda A y_{n+1}\right)-Q_{C}\left(y_{n}-\lambda A y_{n}\right)\right) \\
& \quad+\left(\delta_{n+1}-\delta_{n}\right)\left(S x_{n}-Q_{C}\left(y_{n}-\lambda A y_{n}\right)\right) \| \\
\leq & \delta_{n+1}\left\|\left(S x_{n+1}-S x_{n}\right)\right\|+\left(1-\delta_{n+1}\right)\left\|Q_{C}\left(y_{n+1}-\lambda A y_{n+1}\right)-Q_{C}\left(y_{n}-\lambda A y_{n}\right)\right\| \\
& \quad+\left|\delta_{n+1}-\delta_{n}\right| \cdot\left\|S x_{n}-Q_{C}\left(y_{n}-\lambda A y_{n}\right)\right\| \\
\leq & \delta_{n+1}\left\|x_{n+1}-x_{n}\right\|+\left(1-\delta_{n+1}\right)\left\|y_{n+1}-y_{n}\right\|+\left|\delta_{n+1}-\delta_{n}\right| \cdot\left\|S x_{n}-Q_{C}\left(y_{n}-\lambda A y_{n}\right)\right\| \\
= & \delta_{n+1}\left\|x_{n+1}-x_{n}\right\|+\left(1-\delta_{n+1}\right)+\left\|Q_{C}\left(x_{n+1}-\lambda B x_{n+1}\right)-Q_{C}\left(x_{n}-\lambda B x_{n}\right)\right\| \\
& \quad+\left|\delta_{n+1}-\delta_{n}\right| \cdot\left\|S x_{n}-Q_{C}\left(y_{n}-\lambda A y_{n}\right)\right\| \\
\leq & \delta_{n+1}\left\|x_{n+1}-x_{n}\right\|+\left(1-\delta_{n+1}\right)\left\|x_{n+1}-x_{n}\right\|+\left|\delta_{n+1}-\delta_{n}\right| \cdot\left\|S x_{n}-Q_{C}\left(y_{n}-\lambda_{n} A y_{n}\right)\right\| \\
\leq & \left\|x_{n+1}-x_{n}\right\|+\left|\delta_{n+1}-\delta_{n}\right| M,
\end{aligned}
$$

where $M$ is an appropriate constant such that

$$
M \geq \sup _{n \geq 1}\left\|S x_{n}-Q_{C}\left(y_{n}-\lambda_{n} A y_{n}\right)\right\|
$$

Step 3 . We prove that $\lim _{(n \rightarrow \infty)}\left\|x_{n+1}-x_{n}\right\|=0$.

Setting $w_{n}=\left(x_{(n+1)}-\beta_{n} x_{n}\right) /\left(1-\beta_{n}\right)$ for each $n \geq 1$, we see that

$$
x_{n+1}=\left(1-\beta_{n}\right) w_{n}+\beta_{n} x_{n}, \quad \forall n \geq 1 .
$$


Now, we compute $\left\|w_{n+1}-w_{n}\right\|$ from

$$
\begin{aligned}
\left\|w_{n+1}-w_{n}\right\| & =\left\|\frac{\alpha_{n+1} u+\gamma_{n+1} t_{n+1}}{1-\beta_{n+1}}-\frac{\alpha_{n} u+\gamma_{n} t_{n}}{1-\beta_{n}}\right\| \\
& =\left\|\frac{\alpha_{n+1}}{1-\beta_{n+1}} u+\frac{1-\beta_{n+1}-\alpha_{n+1}}{1-\beta_{n+1}} t_{n+1}-\frac{\alpha_{n}}{1-\beta_{n}} u-\frac{1-\beta_{n}-\alpha_{n}}{1-\beta_{n}} t_{n}\right\| \\
& =\left\|\frac{\alpha_{n+1}}{1-\beta_{n+1}}\left(u-t_{n+1}\right)+\frac{\alpha_{n}}{1-\beta_{n}}\left(t_{n}-u\right)+t_{n+1}-t_{n}\right\| \\
& \leq \frac{\alpha_{n+1}}{1-\beta_{n+1}}\left\|u-t_{n+1}\right\|+\frac{\alpha_{n}}{1-\beta_{n}}\left\|t_{n}-u\right\|+\left\|t_{n+1}-t_{n}\right\| .
\end{aligned}
$$

Combining (3.6) and (3.9), we arrive at

$$
\left\|w_{n+1}-w_{n}\right\|-\left\|x_{n+1}-x_{n}\right\| \leq \frac{\alpha_{n+1}}{1-\beta_{n+1}}\left\|u-t_{n+1}\right\|+\frac{\alpha_{n}}{1-\beta_{n}}\left\|t_{n}-u\right\|+\left|\delta_{n+1}-\delta_{n}\right| M_{1}
$$

It follows from the conditions (1.3), (1.4), and (1.5) that

$$
\limsup _{n \rightarrow \infty}\left(\left\|w_{n+1}-w_{n}\right\|-\left\|x_{n+1}-x_{n}\right\|\right) \leq 0
$$

Hence, by Lemma 2.3 , we obtain that $\lim _{(n \rightarrow \infty)}\left\|w_{n}-x_{n}\right\|=0$. Consequently,

$$
\lim _{n \rightarrow \infty}\left\|x_{n+1}-x_{n}\right\|=\lim _{n \rightarrow \infty}\left(1-\beta_{n}\right)\left\|w_{n}-x_{n}\right\|=0
$$

On the other hand, it follows from the algorithm (3.1) that

$$
x_{n+1}-x_{n}=\alpha_{n}\left(u-x_{n}\right)+\gamma_{n}\left(t_{n}-x_{n}\right) .
$$

From the condition (1.3) and formula (3.12), we see that

$$
\lim _{n \rightarrow \infty}\left\|t_{n}-x_{n}\right\|=0
$$

Step 4 . We prove that $\lim _{(n \rightarrow \infty)}\left\|x_{n}-V x_{n}\right\|=0$. Define a mapping $V: C \rightarrow C$ by

$$
V x=\delta S x+(1-\delta) Q_{C}\left(Q_{C}(I-\mu B)-\lambda A Q_{C}(I-\mu B)\right) x, \quad \forall x \in C,
$$


where $\lim _{(n \rightarrow \infty)} \delta_{n}=\delta \in(0,1)$. From Lemma 2.4, we see that $V$ is a nonexpansive mapping with

$$
\begin{aligned}
F(V) & =F(S) \bigcap F\left(Q_{C}\left(Q_{C}(I-\mu B)-\lambda A Q_{C}(I-\mu B)\right)\right) \\
& =F(S) \bigcap F\left(Q_{C}(I-\lambda A) Q_{C}(I-\mu B)\right) \\
& =F(S) \bigcap F(G) \\
& =F .
\end{aligned}
$$

On the other hand, we have

$$
\begin{aligned}
\left\|x_{n}-V x_{n}\right\| \leq & \left\|x_{n}-x_{n+1}\right\|+\left\|x_{n+1}-V x_{n}\right\| \\
\leq & \left\|x_{n}-x_{n+1}\right\|+\alpha_{n}\left\|u_{n}-V x_{n}\right\|+\beta_{n}\left\|x_{n}-V x_{n}\right\|+\gamma_{n}\left\|t_{n}-V x_{n}\right\| \\
= & \left\|x_{n}-x_{n+1}\right\|+\alpha_{n}\left\|u_{n}-V x_{n}\right\|+\beta_{n}\left\|x_{n}-V x_{n}\right\| \\
& \quad+\gamma_{n}\left\|\delta_{n} S x_{n}+\left(1-\delta_{n}\right) Q_{C}\left(y_{n}-\lambda A y_{n}\right)-\delta S x_{n}-(1-\delta) Q_{C}\left(y_{n}-\lambda A y_{n}\right)\right\| \\
\leq & \left\|x_{n}-x_{n+1}\right\|+\alpha_{n}\left\|u_{n}-V x_{n}\right\|+\beta_{n}\left\|x_{n}-V x_{n}\right\| \\
& \quad+\gamma_{n}\left|\delta_{n}-\delta\right| \cdot\left\|S x_{n}-Q_{C}\left(y_{n}-\lambda_{n} y_{n}\right)\right\| \\
\leq & \left\|x_{n}-x_{n+1}\right\|+\alpha_{n}\left\|u_{n}-V x_{n}\right\|+\beta_{n}\left\|x_{n}-V x_{n}\right\|+\gamma_{n}\left|\delta_{n}-\delta\right| M .
\end{aligned}
$$

This implies that

$$
\left(1-\beta_{n}\right)\left\|x_{n}-V x_{n}\right\| \leq\left\|x_{n}-x_{n+1}\right\|+\alpha_{n}\left\|u_{n}-V x_{n}\right\|+\gamma_{n}\left|\delta_{n}-\delta\right| M
$$

It follows from the conditions (1.3), (1.4), (1.5), and (3.18) that

$$
\lim _{n \rightarrow \infty}\left\|x_{n}-V x_{n}\right\|=0
$$

Step 5. Next, we show that $\lim \sup _{(n \rightarrow \infty)}\left\langle u-\bar{x}, j\left(x_{n}-\bar{x}\right)\right\rangle \leq 0$.

Let $z_{t}$ be the fixed point of the contraction $z \mapsto t u+(1-t) V z_{t}$, where $t \in(0,1)$. That is, $z_{t}=t u+(1-t) V z_{t}$. It follows that

$$
\left\|z_{t}-x_{n}\right\|=\left\|(1-t)\left(V z_{t}-x_{n}\right)+t\left(u-x_{n}\right)\right\| .
$$


On the other hand, for any $t \in(0,1)$, we see that

$$
\begin{aligned}
\left\|z_{t}-x_{n}\right\|^{2}= & \left\langle(1-t)\left(V z_{t}-x_{n}\right)+t\left(u-x_{n}\right), j\left(z_{t}-x_{n}\right)\right\rangle \\
= & (1-t)\left\langle V z_{t}-x_{n}, j\left(z_{t}-x_{n}\right)\right\rangle+t\left\langle u-x_{n}, j\left(z_{t}-x_{n}\right)\right\rangle \\
= & (1-t)\left\langle V z_{t}-V x_{n}, j\left(z_{t}-x_{n}\right)\right\rangle+(1-t)\left\langle V x_{n}-x_{n}, j\left(z_{t}-x_{n}\right)\right\rangle \\
& +t\left\langle u-z_{t}, j\left(z_{t}-x_{n}\right)\right\rangle+t\left\langle z_{t}-x_{n}, j\left(z_{t}-x_{n}\right)\right\rangle \\
\leq & (1-t)\left\|z_{t}-x_{n}\right\|^{2}+(1-t)\left\|V x_{n}-x_{n}\right\| \cdot\left\|z_{t}-x_{n}\right\| \\
& +t\left\langle u-z_{t}, j\left(z_{t}-x_{n}\right)\right\rangle+t\left\|z_{t}-x_{n}\right\|^{2} \\
\leq & \left\|z_{t}-x_{n}\right\|^{2}+\left\|V x_{n}-x_{n}\right\| \cdot\left\|z_{t}-x_{n}\right\|+t\left\langle u-z_{t}, j\left(z_{t}-x_{n}\right)\right\rangle .
\end{aligned}
$$

It follows that

$$
\left\langle z_{t}-u, j\left(z_{t}-x_{n}\right)\right\rangle \leq \frac{1}{t}\left\|V x_{n}-x_{n}\right\| \cdot\left\|z_{t}-x_{n}\right\|, \quad \forall t \in(0,1) .
$$

In view of (3.19), we see that

$$
\limsup _{n \rightarrow \infty}\left\langle z_{t}-u, j\left(z_{t}-x_{n}\right)\right\rangle \leq 0
$$

On the other hand, we see that $Q_{F(V)} u=\lim _{(t \rightarrow 0)} z_{t}$ and $F(V)=F$. It follows that $z_{t} \rightarrow \bar{x}=$ $Q_{F} u$ as $t \rightarrow 0$. Owing to the fact that $j$ is strong to weak* uniformly continuous on bounded subsets of $E$, we see that

$$
\begin{aligned}
\lim _{t \rightarrow 0}\left|\left\langle u-\bar{x}, j\left(x_{n}-\bar{x}\right)\right\rangle-\left\langle z_{t}-u, j\left(z_{t}-x_{n}\right)\right\rangle\right| \leq & \left|\left\langle u-\bar{x}, j\left(x_{n}-\bar{x}\right)\right\rangle-\left\langle u-\bar{x}, j\left(x_{n}-z_{t}\right)\right\rangle\right| \\
& +\left|\left\langle u-\bar{x}, j\left(x_{n}-z_{t}\right)\right\rangle-\left\langle z_{t}-\bar{x}, j\left(z_{t}-x_{n}\right)\right\rangle\right| \\
\leq & \left|\left\langle u-\bar{x}, j\left(x_{n}-\bar{x}\right)-j\left(x_{n}-z_{t}\right)\right\rangle\right| \\
& +\left|\left\langle z_{t}-\bar{x}, j\left(x_{n}-z_{t}\right)\right\rangle\right| \\
\leq & \|u-\bar{x}\| \cdot\left\|j\left(x_{n}-\bar{x}\right)-j\left(x_{n}-z_{t}\right)\right\| \\
& +\left\|z_{t}-\bar{x}\right\| \cdot\left\|x_{n}-z_{t}\right\| \\
= & 0 .
\end{aligned}
$$

Hence, for any $\varepsilon>0$, there exists $\delta>0$ such that for all $t \in(0, \delta)$, the following inequality holds:

$$
\left\langle u-\bar{x}, j\left(x_{n}-\bar{x}\right)\right\rangle \leq\left\langle z_{t}-u, j\left(z_{t}-x_{n}\right)\right\rangle+\varepsilon
$$


Since $\varepsilon$ is arbitrary and (3.23), we see that

$$
\limsup _{n \rightarrow \infty}\left\langle u-\bar{x}, j\left(x_{n}-\bar{x}\right)\right\rangle \leq 0
$$

Step 6. Finally, we show that $x_{n} \rightarrow \bar{x}$ as $n \rightarrow \infty$. Observe that

$$
\begin{aligned}
\left\|x_{n+1}-\bar{x}\right\|^{2} & =\left\langle\alpha_{n} u+\beta_{n} x_{n}+\gamma_{n} t_{n}-\bar{x}, j\left(x_{n+1}-\bar{x}\right)\right\rangle \\
& =\alpha_{n}\left\langle u-\bar{x}, j\left(x_{n+1}-\bar{x}\right)\right\rangle+\beta_{n}\left\langle x_{n}-\bar{x}, j\left(x_{n+1}-\bar{x}\right)\right\rangle+\gamma_{n}\left\langle t_{n}-\bar{x}, j\left(x_{n+1}-\bar{x}\right)\right\rangle \\
& \leq \alpha_{n}\left\langle u-\bar{x}, j\left(x_{n+1}-\bar{x}\right)\right\rangle+\beta_{n}\left\|x_{n}-\bar{x}\right\| \cdot\left\|x_{n+1}-\bar{x}\right\|+\gamma_{n}\left\|t_{n}-\bar{x}\right\| \cdot\left\|\left(x_{n+1}-\bar{x}\right)\right\| \\
& \leq \alpha_{n}\left\langle u-\bar{x}, j\left(x_{n+1}-\bar{x}\right)\right\rangle+\beta_{n}\left\|x_{n}-\bar{x}\right\| \cdot\left\|x_{n+1}-\bar{x}\right\|+\gamma_{n}\left\|x_{n}-\bar{x}\right\| \cdot\left\|\left(x_{n+1}-\bar{x}\right)\right\| \\
& =\alpha_{n}\left\langle u-\bar{x}, j\left(x_{n+1}-\bar{x}\right)\right\rangle+\left(1-\alpha_{n}\right)\left\|x_{n}-\bar{x}\right\| \cdot\left\|x_{n+1}-\bar{x}\right\| \\
& \leq \alpha_{n}\left\langle u-\bar{x}, j\left(x_{n+1}-\bar{x}\right)\right\rangle+\frac{1-\alpha_{n}}{2}\left(\left\|x_{n}-\bar{x}\right\|^{2}+\left\|x_{n+1}-\bar{x}\right\|^{2}\right)
\end{aligned}
$$

which implies that

$$
\left\|x_{n+1}-x_{n}\right\|^{2} \leq\left(1-\alpha_{n}\right)\left\|x_{n}-\bar{x}\right\|^{2}+2 \alpha_{n}\left\langle u-\bar{x}, j\left(x_{n+1}-\bar{x}\right)\right\rangle .
$$

From the conditions (1.3) and (3.26) and applying Lemma 2.5 to (3.28), we obtain that

$$
\lim _{n \rightarrow \infty}\left\|x_{n}-\bar{x}\right\|=0
$$

This completes the proof.

Remark 3.2. Since $L^{p}$ for all $p \geq 2$ is uniformly convex and 2 uniformly smooth, we see that Theorem 3.1 is applicable to $L^{p}$ for all $p \geq 2$. There are a number of sequences satisfying the restrictions (C1)-(C3), for example, $\alpha_{n}=1 /(n+1), \beta_{n}=n /(2 n+1), \gamma_{n}=n^{2} /\left(2 n^{2}+3 n+1\right)$ for each $n \geq 1$.

Corollary 3.3. Let $H$ be a real Hilbert space and $C$ a nonempty closed convex subset of $H$. Let $A, B: C \rightarrow H$ be $\alpha$-inverse strongly monotone mapping and $\beta$-inverse strongly monotone mapping, respectively, and $S: C \rightarrow C$ nonexpansive mappings with a fixed point. Assume that $F=F(S) \bigcap F\left(G^{\prime}\right) \neq \emptyset$, where $G^{\prime}=P_{C}\left[P_{C}(x-\mu B x)-\lambda A P_{C}(x-\mu B x)\right]$. Suppose that $\left\{x_{n}\right\}$ is generated by

$$
\begin{gathered}
x_{1}=u \in C, \\
y_{n}=P_{C}\left(x_{n}-\mu B x_{n}\right), \\
x_{n+1}=\alpha_{n} u+\beta_{n} x_{n}+\gamma_{n}\left[\delta_{n} S x_{n}+\left(1-\delta_{n}\right) P_{C}\left(y_{n}-\lambda A y_{n}\right)\right], \quad n \geq 1,
\end{gathered}
$$


where $\delta_{n} \subset[0,1], \lambda \in(0,2 \alpha), \mu \in(0,2 \beta)$ and $\left\{\alpha_{n}\right\},\left\{\beta_{n}\right\}$, and $\left\{\gamma_{n}\right\}$ are three sequences in $(0,1)$, and the following conditions are satisfied:

(1) $\alpha_{n}+\beta_{n}+\gamma_{n}=1$, for all $n \geq 1$,

(2) $\lim _{(n \rightarrow \infty)} \alpha_{n}=0, \sum_{n=0}^{\infty} \alpha_{n}=\infty$,

(3) $0<\liminf _{(n \rightarrow \infty)} \beta_{n} \leq \limsup \sup _{(n \rightarrow \infty)} \beta_{n}<1$,

(4) $\lim _{(n \rightarrow \infty)} \delta_{n}=\delta \in(0,1)$.

Then the sequence $\left\{x_{n}\right\}$ defined by (3.30) converges strongly to $\bar{x}=P_{F} u$, and $(\bar{x}, \bar{y})$ is a solution of the problem (1.4), where $\bar{y}=P_{C}(\bar{x}-\mu B \bar{x}), P_{C}$ is the projection of $H$ onto $C$, and $P_{F}$ is the projection of $C$ onto $F$.

Remark 3.4. Theorem 3.1 and Corollary 3.3 improve and extend the corresponding results announced by other authors, such as $[2-4,6,8,10-12,14]$.

\section{Acknowledgments}

The authors would like to thank editors and referees for many useful comments and suggestions for the improvement of the paper. This work was partially supported by the Natural Science Foundation of Zhejiang Province (Y6100696) and NSFC (11071169).

\section{References}

[1] G. Stampacchia, "Formes bilinéaires coercitives sur les ensembles convexes," Comptes Rendus de l'Académie des Sciences, vol. 258, pp. 4413-4416, 1964.

[2] M. A. Noor, "Some algorithms for general monotone mixed variational inequalities," Mathematical and Computer Modelling, vol. 29, no. 7, pp. 1-9, 1999.

[3] Y. Yao and M. A. Noor, “On viscosity iterative methods for variational inequalities," Journal of Mathematical Analysis and Applications, vol. 325, no. 2, pp. 776-787, 2007.

[4] M. A. Noor, "New approximation schemes for general variational inequalities," Journal of Mathematical Analysis and Applications, vol. 251, no. 1, pp. 217-229, 2000.

[5] M. Aslam Noor, "Some developments in general variational inequalities," Applied Mathematics and Computation, vol. 152, no. 1, pp. 199-277, 2004.

[6] R. U. Verma, "Generalized system for relaxed cocoercive variational inequalities and projection methods," Journal of Optimization Theory and Applications, vol. 121, no. 1, pp. 203-210, 2004.

[7] S. S. Chang, H. W. Joseph Lee, and C. K. Chan, "Generalized system for relaxed cocoercive variational inequalities in Hilbert spaces," Applied Mathematics Letters, vol. 20, no. 3, pp. 329-334, 2007.

[8] L.-C. Ceng and J.-C. Yao, "An extragradient-like approximation method for variational inequality problems and fixed point problems," Applied Mathematics and Computation, vol. 190, no. 1, pp. 205215, 2007.

[9] X. Qin, S. M. Kang, and M. Shang, "Generalized system for relaxed cocoercive variational inequalities in Hilbert spaces," Applicable Analysis, vol. 87, no. 4, pp. 421-430, 2008.

[10] H. Iiduka and W. Takahashi, "Strong convergence theorems for nonexpansive mappings and inversestrongly monotone mappings," Nonlinear Analysis. Theory, Methods E Applications, vol. 61, no. 3, pp. 341-350, 2005.

[11] X. Qin, S. Y. Cho, and S. M. Kang, "Convergence of an iterative algorithm for systems of variational inequalities and nonexpansive mappings with applications," Journal of Computational and Applied Mathematics, vol. 233, no. 2, pp. 231-240, 2009.

[12] L.-C. Ceng, C.-y. Wang, and J.-C. Yao, "Strong convergence theorems by a relaxed extragradient method for a general system of variational inequalities," Mathematical Methods of Operations Research, vol. 67 , no. 3, pp. 375-390, 2008.

[13] D. L. Zhu and P. Marcotte, "Co-coercivity and its role in the convergence of iterative schemes for solving variational inequalities," SIAM Journal on Optimization, vol. 6, no. 3, pp. 714-726, 1996. 
[14] Y. Yao, M. Aslam Noor, K. Inayat Noor, Y.-C. Liou, and H. Yaqoob, "Modified extragradient methods for a system of variational inequalities in Banach spaces," Acta Applicandae Mathematicae, vol. 110, no. 3, pp. 1211-1224, 2010.

[15] J. Li, “The generalized projection operator on reflexive Banach spaces and its applications," Journal of Mathematical Analysis and Applications, vol. 306, no. 1, pp. 55-71, 2005.

[16] H. K. Xu, "Inequalities in Banach spaces with applications," Nonlinear Analysis. Theory, Methods $\mathcal{E}$ Applications, vol. 16, no. 12, pp. 1127-1138, 1991.

[17] R. E. Bruck, Jr., "Nonexpansive retracts of Banach spaces," Bulletin of the American Mathematical Society, vol. 76, pp. 384-386, 1970.

[18] T. Suzuki, "Strong convergence of Krasnoselskii and Mann's type sequences for one-parameter nonexpansive semigroups without Bochner integrals," Journal of Mathematical Analysis and Applications, vol. 305, no. 1, pp. 227-239, 2005.

[19] R. E. Bruck, Jr., "Properties of fixed-point sets of nonexpansive mappings in Banach spaces," Transactions of the American Mathematical Society, vol. 179, pp. 251-262, 1973.

[20] S. Reich, "Asymptotic behavior of contractions in Banach spaces," Journal of Mathematical Analysis and Applications, vol. 44, pp. 57-70, 1973. 


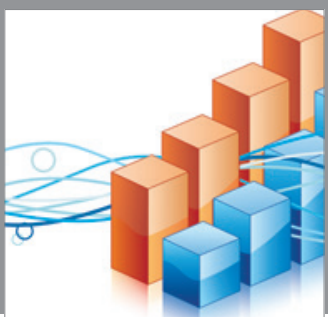

Advances in

Operations Research

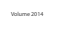

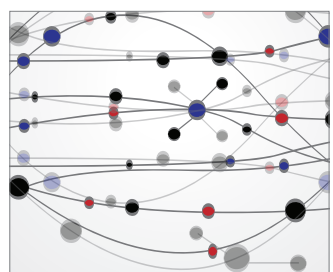

\section{The Scientific} World Journal
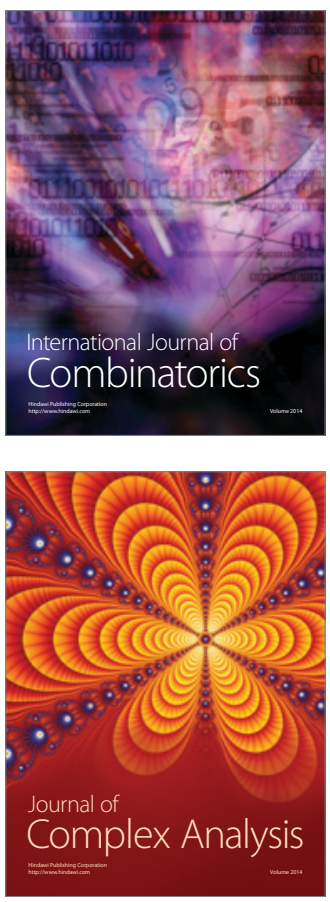

International Journal of

Mathematics and

Mathematical

Sciences
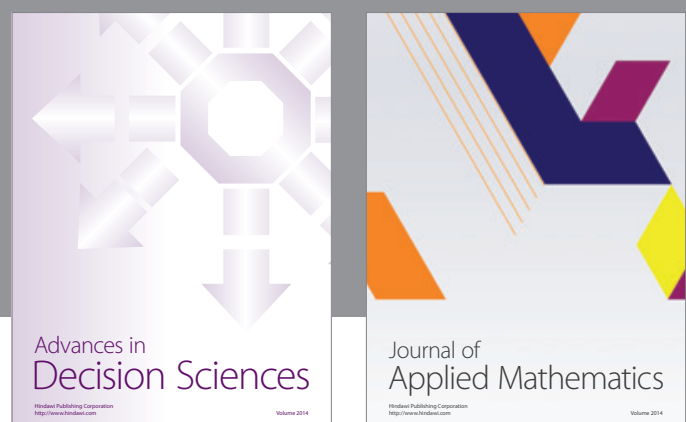

Journal of

Applied Mathematics
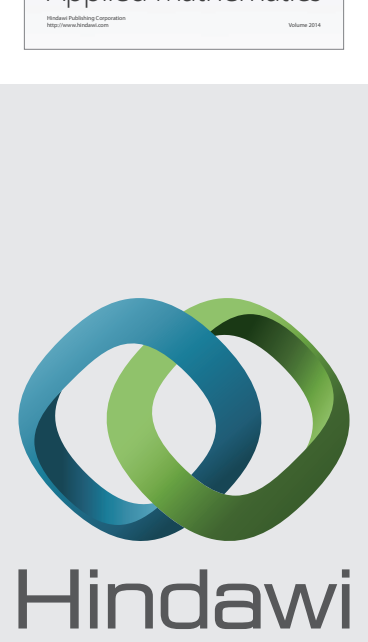

Submit your manuscripts at http://www.hindawi.com
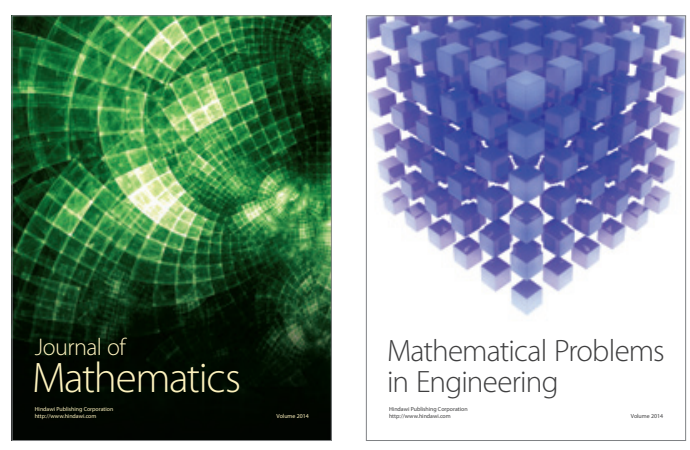

Mathematical Problems in Engineering
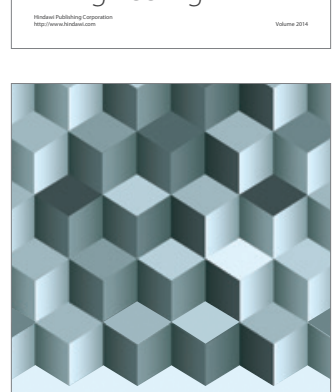

Journal of

Function Spaces
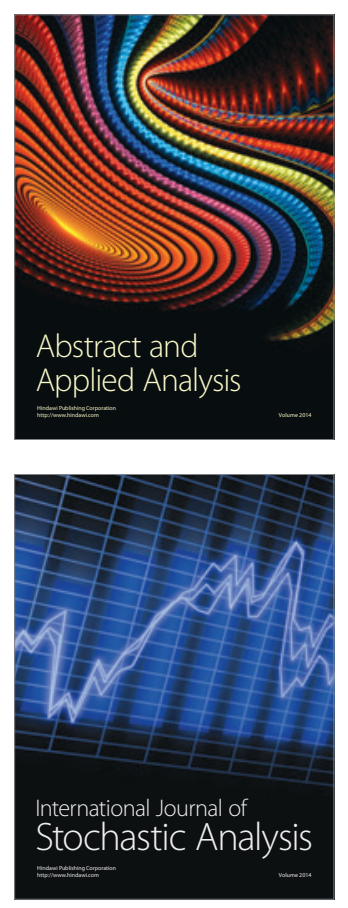

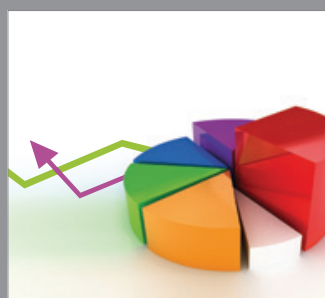

ournal of

Probability and Statistics

Promensencen
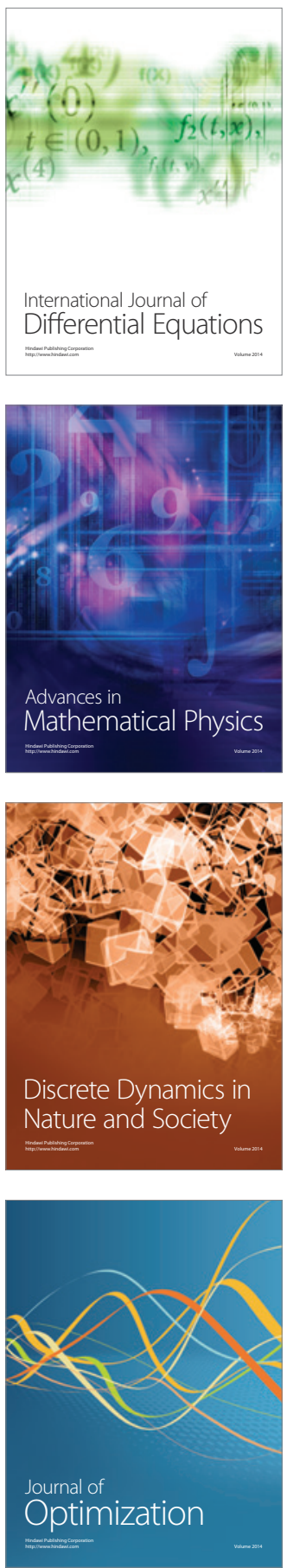